\title{
Predicción del calentamiento global mediante el desarrollo de un modelo de series de tiempo*
}

\section{Predicting Global Warming by Developing a Time Series Model}

\author{
Juan Bacilio Guerrero Escamilla,** Yamile Rangel Martínez,*** \\ Sócrates López Pérez ${ }^{* * *}$
}

Recibido: 2016-08-18 // Aprobado: 2016-10-12// Disponible en línea: 2017-01-30

Cómo citar este artículo: Guerrero Escamilla J. B., Rangel Martínez, Y. y López Pérez, S. (2017). Predicción del calentamiento global mediante el desarrollo de un modelo de series de tiempo. Ambiente y Desarrollo, 21(40), 125-139. https://doi.org/10.11144/Javeriana.ayd21-40.pcgm doi:10.11144/Javeriana.ayd21-40.pcgm

\begin{abstract}
Resumen
Las alteraciones en los ecosistemas debido al cambio climático han ocasionado que se hagan estudios globales sobre la temperatura promedio del planeta. Se desarrolla un modelo probabilístico de series de tiempo para observar la dinámica de la temperatura en el tiempo y determinar el calentamiento global. Se analiza un periodo de 165 años, desde 1850 a 2015, con base en los reportes de temperatura global de la APA de Estados Unidos, y se observa que desde 1963 esta se ha incrementado en $0.29^{\circ} \mathrm{C}$. Sin embargo, es hasta 1996 cuando los efectos negativos sobre el planeta se hacen presentes, ya que la temperatura promedio oscila por arriba de los intervalos establecidos por el modelo. Por otro lado, se realiza una proyección al año 2030, producto de lo cual se obtiene un parámetro entre 14.55 y $16.33^{\circ} \mathrm{C}$.
\end{abstract}

Palabras clave: modelo de series de tiempo; calentamiento global

Este artículo es resultado del proyecto de investigación Predicción de gases efecto invernadero del estado de Hidalgo (México), financiado por la Secretaría de Medio Ambiente y Recursos Naturales (Semarnat), México.

** Doctor en Ciencias Sociales; investigador, Área Académica de Sociología y Demografía de la Universidad Autónoma del Estado de Hidalgo, México. Correo electrónico: guerreroescamilla@yahoo.com.mx

*** Doctora en Ciencias Ambientales; investigadora, Área Académica de Sociología y Demografía, Universidad Autónoma del Estado de Hidalgo. Correo electrónico: yamilerangelm@gmail.com

**** Doctor en Desarrollo Regional; investigador, Área Académica de Sociología y Demografía, Universidad Autónoma del Estado de Hidalgo. Correo electrónico: lopezsoc@gmail.com 


\begin{abstract}
Changes in ecosystems due to climate change have led to global studies on the average temperature of the planet. We developed a probabilistic model of time series to observe the dynamics of temperature over time and determine global warming. We analyzed a 165-year period, from 1850 to 2015, based on the global temperature reports of the APA (in the United States). We could observe that since 1963 temperature has increased by $0.29^{\circ} \mathrm{C}$. However, only until 1996 the adverse effects on the planet became evident, given that the average temperature fluctuates above the intervals established by the model. On the other hand, we projected up to the year 2030, from which we obtained a parameter between 14.55 and $16.33^{\circ} \mathrm{C}$.
\end{abstract}

Keywords: time series model; global warming 


\section{Introducción}

En la actualidad el cambio climático está afectando al planeta, pues se ha hecho presente mediante fenómenos atmosféricos y oceánicos. Para los especialistas en medio ambiente (Panel intergubernamental de cambio climático, IPCC), estos fenómenos han sido resultado del aumento de la temperatura del planeta, ya que en los últimos cien años se ha incrementado en $0.74^{\circ} \mathrm{C}$. Es muy probable que dicho incremento sea resultado de las emisiones de gases de efecto invernadero generadas por las actividades humanas, de las cuales se desglosan las económicas y sociales (GreenFacts, 2007).

Una de las discusiones actuales sobre la crisis ambiental se encuentra enmarcada por la irracionalidad económica (Baran y Sweezy). En ese contexto, la libertad humana ha hecho que cada individuo satisfaga sus intereses mediante la búsqueda de beneficios, sin darse cuenta de los efectos colaterales $o$ externalidades que ha ocasionado en el medio ambiente, pues la acumulación de riqueza en una nación se cuantifica a partir del grado de explotación de los recursos renovables y no renovables.

Para los ecologistas, el crecimiento económico — acumulación de la riqueza mediante el PIBlleva a una sobreexplotación del hábitat. Para las economías emergentes, que pelean por conseguir estatus a la altura de los países desarrollados, hace que el consumo de bienes y servicios se incremente. Por lo tanto, están desarrollando un crecimiento insostenible, es decir, se están aglutinado en lo material, lo cual no es condición precisa para experimentar el desarrollo social (López, 2012).

Como se puede observar, es fundamental que se hagan estudios globales, ya que al existir grandes alteraciones en los ecosistemas es fundamental que se prediga el incremento que va a tener la temperatura promedio, y con ello, ejecutar determinadas medidas que amortigüen los embates del cambio climático global.

Desarrollar un modelo de series tiempo probabilístico sobre el calentamiento global, a partir de la temperatura promedio del planeta, es novedoso, ya que dichos modelos se utilizan para economía e ingeniería financiera. Algunos modelos similares para cuestiones ambientales son los modelos de corte transversal, como la ecuación IPAT. De Barry Commoner, biólogo, profesor universitario y político estadounidense, y sus colegas Paul R. Ehrlich y John Holdren, estudiaron que el impacto humano sobre el medio ambiente es una función del producto de la cantidad de población, el consumo de esta —o de su afluencia- y la tecnología empleada.

El presente modelo de series de tiempo se desarrolla con la finalidad de observar la dinámica de la temperatura promedio en el tiempo y determinar el calentamiento global en periodos de tiempo. Se analizó un periodo de 165 años, de 1850 a 2015, con base en los reportes de temperatura global de la Agencia de Protección al Ambiente (APA) de Estados Unidos.

Se entiende por dinámica de la temperatura, las consecuencias de la emisión de gases a la atmósfera debido a las actividades humanas, lo cual da origen al efecto invernadero. El mecanismo de este último calienta la atmósfera de la tierra, la cual se compone de una delgada capa de gases que rodea al planeta y que son fundamentales para el desarrollo de la mayor parte de la vida. Su composición química se conforma en gran parte por dos gases: nitrógeno en $79 \%$ y oxígeno en $20 \%$, el $1 \%$ restante se forma por diversos gases como argón y dióxido de carbono (Caballero, Lozano y Ortega, 2007).

Para los expertos en medio ambiente, el calentamiento global ha ido de la mano con una tendencia hacia el aumento de dióxido de carbono (C02). Este comportamiento puede estar asociado con procesos naturales, sin embargo, hay un mecanismo humano (actividad humana) explicativo: la tala de árboles y la quema de combustibles fósiles (carbón y petróleo), actividades estas que han ocasionado un aumento del C02 atmosférico, pues al estar por encima del que requiere el planeta, se genera el calentamiento global, cuyo principal indicador es el incremento de la temperatura promedio.

La mayoría de las afirmaciones sobre el calentamiento global se respaldan en la construcción de modelos matemáticos y estadísticos, los cuales asumen que el $\mathrm{CO} 2$ generado por la actividad humana es 
la base fundamental del cambio climático. Los resultados arrojados por estos trabajos de investigación aseguran que duplicar la radiación por gases de efecto invernadero con respecto a lo que se conoce, hace previsible que en el futuro haya más calentamiento que en la actualidad (Ferrero, 2010).

Dichos trabajos también predicen la temperatura en el futuro — de cincuenta a cien años-. La impresión que generan es la de ser una ciencia rigurosa, en la cual la principal aportación es la especulación climática, donde la teoría del calentamiento global es el resultado de la actividad humana (Ferrero, 2010).

Este trabajo tiene la finalidad de construir y desarrollar un modelo probabilístico de series de tiempo sobre el calentamiento global, a partir de un rigor científico, con la premisa de que la dinámica de la temperatura es un fenómeno aleatorio $\mathrm{y}$, por lo tanto, existe incertidumbre sobre su comportamiento.

Asimismo, en esta investigación se hace una comprobación de dicho incremento y, aunado a esto, se pronostica la temperatura promedio del planeta para el año 2030, límite para evaluar las acciones estipuladas en la Agenda 2030 (según el acuerdo de París, que entró en vigor en noviembre de 2016).

Para desarrollar el estado referencial y geográfico del modelo de series de tiempo del calentamiento global, es necesario basarse en la metodología de Box-Jenkins, considerando la demarcación geográfica a escala planetaria. Sus orígenes se encuentran en la década de los setenta, y sus principales precursores fueron G. P. E. Box y G. M. Jenkins. El objetivo central de esta metodología es crear un modelo estadístico que pronostique el comportamiento de un fenómeno — social, económico o ambiental, entre otros - en el tiempo, tomando como herramienta la inferencia estadística (Rosales, 2009).

El calentamiento global se expresaría de la siguiente forma:

$$
\mathrm{Tp}=\mathrm{f}(\mathrm{t})=\beta_{0}+\beta_{1} \mathrm{t}+\mathrm{u}_{\mathrm{i}}
$$

Donde:

- Tp es la temperatura promedio global

- $\quad \beta_{0}$ es la temperatura promedio global cuando el tiempo es constante

- $\quad \beta_{1}$ es tasa de cambio instantánea que experimenta la temperatura promedio global por cada año trascurrido

- $\quad t$ es tiempo en años (1850-2015)

- $\mathrm{u}_{\mathrm{i}}$ es el margen de error que no puede ser explico por el modelo

A partir de esta expresión algebraica, el modelo de series tiempo estará determinado por un objetivo general, una justificación y sus alcances y límites.

En el contexto anterior, el objetivo general de este trabajo es pronosticar la dinámica de la temperatura promedio del planeta, a partir de los registros de cambio climático global de la Agencia de Protección del Ambiente de los Estados Unidos, en el periodo de 1850 a 2015. En cuanto a los alcances y limitaciones de esta investigación, se encuentran los siguientes:

Alcances

- Mediante el desarrollo del modelo de series de tiempo se podrá pronosticar el incremento de la temperatura durante los últimos cien ańos.

- Se obtendrá la predicción de la temperatura promedio del planeta para el año 2030.

Limitaciones

- La información obtenida de la Agencia de Protección del Ambiente de los Estados Unidos en el periodo de 1850 a 2015 es anual, lo cual solamente proporciona 165 observaciones. Lo deseable sería que se obtuviera información mensual de esos 165 años, con ello se tendría una mejor representatividad del fenómeno. 
- Sería recomendable, posteriormente, construir un modelo de datos panel, ya que se podrían incluir otras variables que influyen en la dinámica de la temperatura promedio del planeta, pues el tomar solamente el tiempo como variable independiente no da mucha certeza de la dinámica del fenómeno.

En síntesis, el desarrollo de este modelo de series tiempo de la temperatura promedio del planeta únicamente va a pronosticar la dinámica del calentamiento global en el tiempo.

\section{Metodología del modelo de Box-Jenkins}

Las etapas que se deben seguir en la construcción de un modelo de Box-Jenkins son las siguientes (Rosales, 2009):

- 1. a etapa: se detecta el tipo de proceso estocástico que han de seguir los datos. Esto conlleva formar una serie estacionaria, para lo cual se efectúan las pruebas de estacionalidad a la serie original. En caso de que esta no sea estacionaria, la variable en estudio puede diferenciarse $n$ veces, hasta que lo sea.

- 2. etapa: en esta etapa se estiman los coeficientes de los términos autorregresivos y de media móvil incluidos en el modelo. Algunas veces la estimación se efectúa por medio del método de mínimos cuadrados o máxima verosimilitud.

- 3. a etapa: se evalúa la eficiencia del modelo de series tiempo, lo cual se realiza mediante el análisis de los coeficientes o parámetros del modelo, la evaluación de la bondad de ajuste y el análisis de los residuales.

- Análisis de los coeficientes: el modelo estimado debe cumplir con las condiciones de estacionaridad e invertibilidad y que exista significancia estadística en los rezagos incorporados.

- Bondad de ajuste: es fundamental identificar cuál de los modelos presenta mejor ajuste. Para hacer esa verificación se utiliza $\mathrm{R}^{2}$ ajustado, el Drubin-Watson y el Akaike.

- Análisis de los residuales: se grafican los residuales en función del tiempo, con la finalidad de que sean homocedásticos y que tengan varianza constante. Para esto se hacen pruebas de autocorrelación serial de orden superior y de heterocedasticidad.

- 4. ${ }^{a}$ etapa: se predice un periodo futuro a partir del modelo seleccionado, es decir, aquel que es mejor resultante de las etapas anteriores.

El área geográfica del modelo de series de tiempo sobre el calentamiento global está enmarcada por el planeta. La delimitación temporal del presente trabajo de investigación está demarcada en un periodo de 165 años (1850 a 2015). Los datos que proporciona la organización de cambio climático global de la Agencia de Protección del Ambiente de los Estados Unidos son anuales. Por ende, el modelo de series de tiempo de la temperatura promedio del planeta es anual.

\section{Modelamiento del calentamiento global}

\section{Identificación y selección del modelo}

En este apartado se debe seleccionar el modelo más adecuado para pronosticar la serie de tiempo, lo cual se hace mediante la evaluación de la estacionaridad. Esta debe cumplir con lo siguiente (Villavicencio, 2008):

- Media: $E\left(Y_{t}\right)=\mu$

- Varianza: $\operatorname{var}\left(\mathrm{Y}_{\mathrm{t}}\right)=\mathrm{E}\left(\mathrm{Y}_{\mathrm{t}}-\mu\right)^{2}=\sigma^{2}$

- Covarianza: $\gamma_{\mathrm{t}}=\mathrm{E}\left[\left(\mathrm{X}_{\mathrm{t}}-\mu\right)\left(\mathrm{X}_{\mathrm{t}+1}-\mu\right)\right]$

El objetivo central es validar que la serie no presenta tendencia, lo cual se realiza mediante tres herramientas: la primera, a través de la visualización del gráfico de la serie; la segunda, con los correlogramas; y la tercera, con la prueba de raíz unitaria (Dichey-Fuller o Phillips-Perron). 
Para el análisis de los datos se utilizó el software EViews, un paquete estadístico de lenguaje propio, para Microsoft Windows, usado principalmente para análisis econométrico y que ha sido desarrollado por Quantitative Micro Software (QMS).

Con base en lo anterior, el comportamiento de la serie se muestra en la figura 1.

Con base en la figura 1, se puede observar que la serie de tiempo tiene tendencia. Por tanto, existe la sospecha de no estacionaridad. Esto también se puede corroborar mediante el correlograma de la temperatura de la tabla 1, en la cual se puede ver que la serie tiene un decrecimiento suavizado, lo que quiere decir que no tiene estacionaridad.

A partir de la tabla 1, una opción más formal es mediante la prueba de raíces unitarias de DickeyFuller. En el recuadro de las raíces unitarias se puede observar que con un P-valor de 0.9652 no hay suficiente evidencia estadística para rechazar la hipótesis nula. Por tanto, hay raíz unitaria y no existe estacionaridad. Mediante una serie de diferenciaciones, el modelo más adecuado es el Arima.

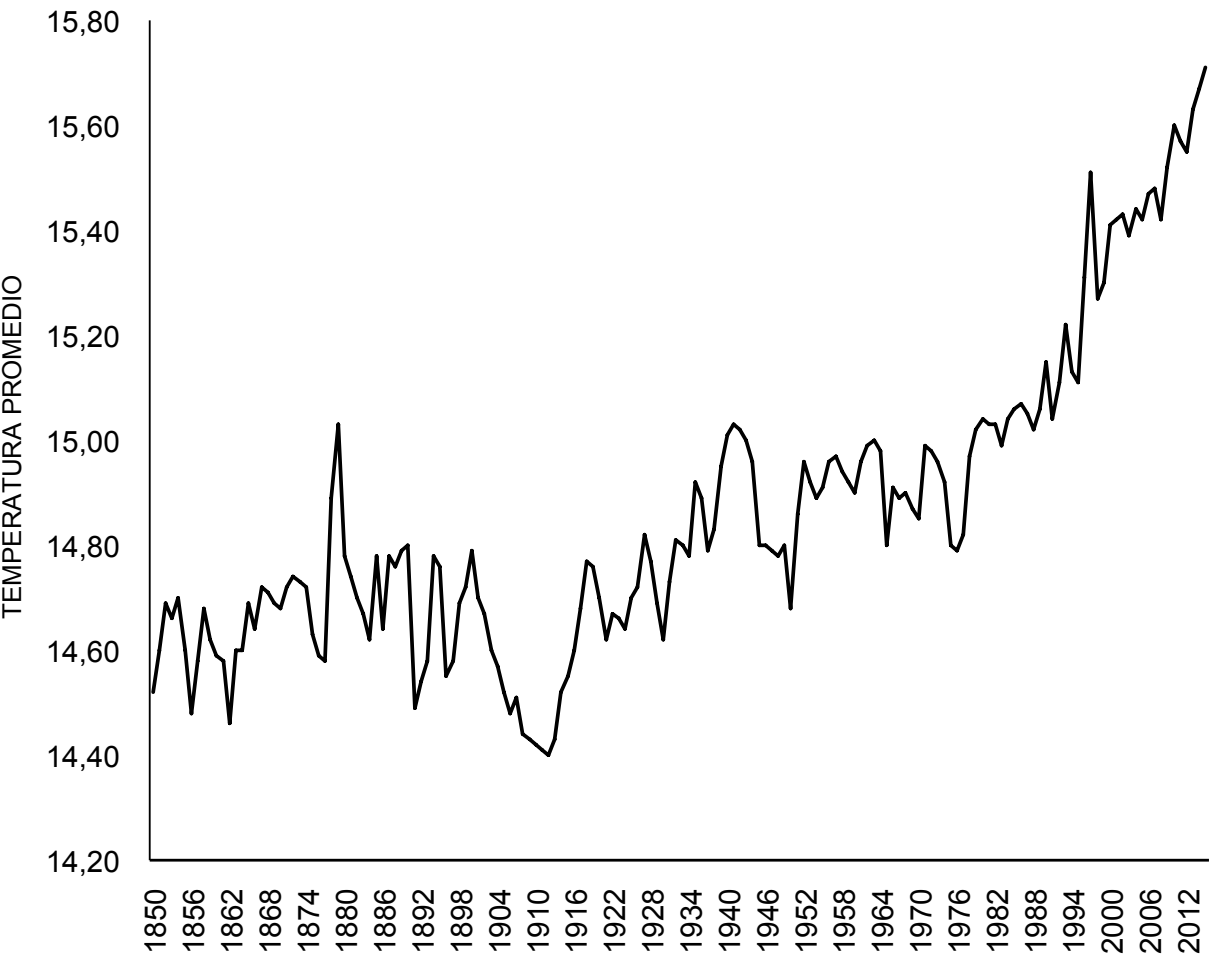

Figura 1. Comportamiento de la serie de tiempo del calentamiento global

Fuente: Agencia de Protección del Ambiente de los Estados Unidos 
Tabla 1. Primera corrida de series de tiempo

Correlogram of TEMPERATURA

Data: $08 / 24 / 16$

Sample: $1850-2015$

Inculded observations: 166

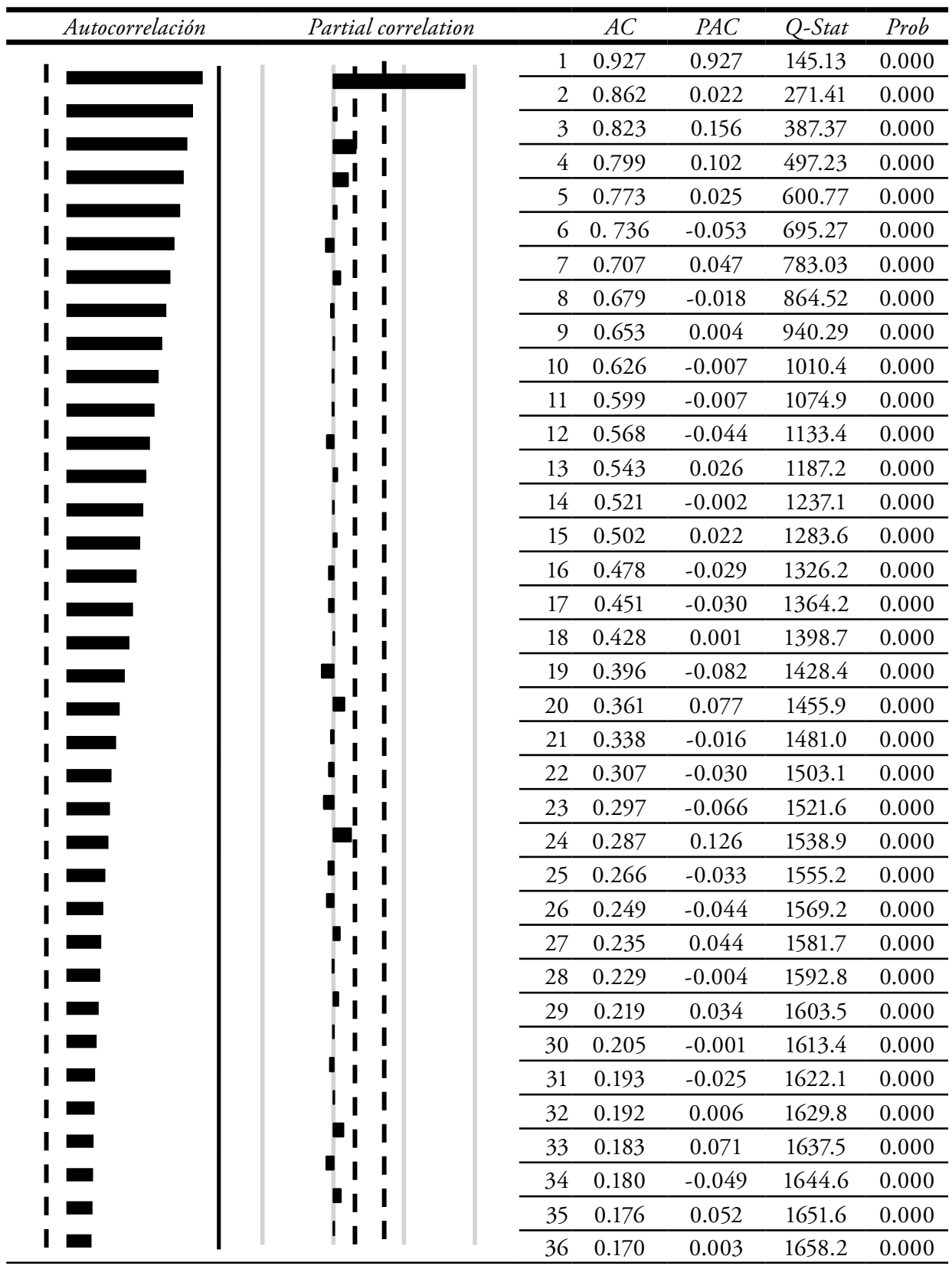

Fuente: elaboración propia 
Null Hypothesis: TEMPERATURA has a unit root

Exogenous: Constant

Lag Length: 2 (Automatic-based on SIC, maxlag=13)

\begin{tabular}{llcl}
\hline & & & \\
\hline & & & \\
\hline & & & \\
Augmented & & \\
\hline Test critical values: & test statistic & 0.104479 & 0.9652 \\
& $1 \%$ level & -3.470679 & \\
& $5 \%$ level & -2.879155 & \\
& $10 \%$ level & -2.576241 & \\
\hline
\end{tabular}

*MacKinnon (1996) one-sided p-values.

Augmented Dickey-Fuller Test Equation

Dependent Variable: D(TEMPERATURA)

Method: Least Squares

Date: 08/24/16

Time: 11:31

Included observations: 163 after adjustments

\begin{tabular}{llcrr} 
Variable & Coefficient & Std. Error & t-Statistic & Prob. \\
TEMPERATURA(-1) & 0.002525 & 0.024170 & 0.104479 & 0.9169 \\
D(TEMPERATURA(-1)) & -0.075290 & 0.078597 & -0.957931 & 0.3396 \\
D(TEMPERATURA(-2)) & -0.266787 & 0.077956 & -3.422278 & 0.0008 \\
C & -0.028954 & 0.358958 & -0.080661 & 0.9358 \\
& & & & \\
R-squared & 0.074171 & Mean dependent var & 0.006258 \\
Adjusted R-squared & 0.056703 & S.D. dependent var & 0.085122 \\
S.E. of regression & 0.082674 & Akaike info criterion & -2.123598 \\
Sum squared resid & 1.086754 & Schwarz criterion & -2.047678 \\
Log likelihood & 177.070 & Hannan-Quinn criter. & -2.092775 \\
F-statistic & 4.245994 & Durbin-Watson stat & 2.087012 \\
Prob(F-statistic) & 0.006448 & & \\
\hline
\end{tabular}

Fuente: elaboración propia 
Tabla 2. Análisis de coeficientes del modelo Arima

Correlogram of Residuals

Date: 08/25/16 Time: 10:06

Sample: 18502015

Included observations: 131

Q-statistic probabilities adjusted for 3 ARMA terms

\begin{tabular}{|c|c|c|c|c|c|c|c|c|c|c|}
\hline \multicolumn{3}{|c|}{ Autocorrelation } & \multicolumn{3}{|c|}{ Autocorrelation } & & \multirow{2}{*}{$\frac{A C}{-0.058}$} & \multirow{2}{*}{$\frac{P A C}{-0.058}$} & \multirow{2}{*}{$\frac{Q-S t a t}{0.4494}$} & \multirow[t]{2}{*}{ Prob } \\
\hline I & & I & 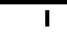 & & I & 1 & & & & \\
\hline I & - & I & I & - & I & 2 & 0.140 & 0.137 & 3.1066 & \\
\hline i & & i & i & 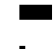 & i & 3 & 0.008 & 0.024 & 3.1155 & \\
\hline I & 1 & I & I & I & I & 4 & -0.026 & -0.045 & 3.2080 & 0.073 \\
\hline I & $\mathbf{I}$ & I & i & च & I & 5 & 0.204 & 0.201 & 8.9816 & 0.011 \\
\hline I & & 1 & I & & I & 6 & -0.222 & -0.204 & 15.7020 & 0.001 \\
\hline In & & I & I & & I & 7 & 0.113 & 0.052 & 17.5030 & 0.002 \\
\hline I & - & I & I & - & i & 8 & -0.052 & 0.007 & 17.8810 & 0.003 \\
\hline I & - & I & I & । & I & 9 & -0.086 & -0.114 & 18.9430 & 0.004 \\
\hline I & & i & i & $\boldsymbol{E}$ & i & 10 & 0.094 & 0.065 & 20.2130 & 0.005 \\
\hline I & - & I & I & - & I & 11 & -0.154 & -0.054 & 23.6610 & 0.003 \\
\hline I & & i & I & - & I & 12 & 0.094 & -0.004 & 24.9640 & 0003 \\
\hline i & घ & I & I & I & I & 12 & & & & 0.005 \\
\hline I & 1 & I & I & 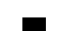 & I & 13 & 0.011 & 0.098 & 24.9820 & 0.005 \\
\hline I & 1 & I & I & i & I & 14 & 0.002 & -0.001 & 24.9830 & 0.009 \\
\hline I & 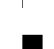 & i & I & 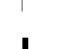 & I & 15 & 0.092 & 0.025 & 26.2620 & 0.010 \\
\hline I & 1 & I & I & 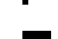 & I & 16 & 0.017 & 0.119 & 26.3070 & 0.015 \\
\hline I & 1 & I & I & - & I & 17 & 0.186 & 0.101 & 31.5690 & 0.005 \\
\hline I & 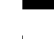 & I & I & D & I & 18 & 0.002 & 0.001 & 31.5690 & 007 \\
\hline i & I & I & I & I & i & 10 & & & & 0.007 \\
\hline I & I & I & I & I & I & 19 & 0.040 & 0.038 & 31.8220 & 0.011 \\
\hline I & च & I & I & | & I & 20 & 0.072 & 0.024 & 32.6390 & 0.013 \\
\hline I & E & I & I & 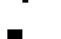 & I & 21 & -0.067 & -0.063 & 33.3530 & 0.015 \\
\hline i & - & I & I & I & I & 22 & 0.058 & 0.014 & 33.8820 & 0.019 \\
\hline I & E & I & I & 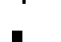 & I & 23 & -0.090 & -0.043 & 35.1860 & 0.019 \\
\hline I & । & I & 1 & I & I & 24 & 0.002 & -0.025 & 35.1870 & 0.027 \\
\hline i & 1 & I & I & 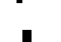 & i & 25 & 0.010 & 0.039 & 35.2030 & 0.037 \\
\hline I & 1 & I & I & 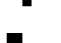 & I & 26 & & -0.065 & & 0033 \\
\hline I & 口 & I & I & 口 & 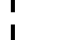 & 20 & -0.102 & -0.005 & 30.9500 & 0.033 \\
\hline I & I & I & I & 口 & i & 27 & -0.020 & -0.079 & 37.0030 & 0.044 \\
\hline i & [ & I & I & $I$ & I & 28 & -0.102 & -0.017 & 38.7630 & 0.039 \\
\hline I & I & I & I & 1 & I & 29 & 0.040 & 0.005 & 39.0310 & 0.048 \\
\hline I & $\mathbf{I}$ & i & i & I & I & 30 & -0.019 & -0.037 & 39.0910 & 0.062 \\
\hline I & - & I & I & I & I & 31 & -0.066 & -0.030 & 39.8390 & 0.068 \\
\hline I & I & I & 1 & I & I & 32 & 0.045 & -0.031 & 40.1950 & 0.081 \\
\hline I & I & I & I & घ & I & 33 & 0.039 & 0.063 & 40.4620 & 0.096 \\
\hline I & 1 & 1 & I & I & I & 34 & 0.024 & -0.031 & 40.5660 & 0.117 \\
\hline i & 1 & i & i & $\mathbf{I}$ & i & 35 & -0.006 & -0.027 & 40.5730 & 0142 \\
\hline I & च & I & I & n & I & 3) & 002 & & & 0.142 \\
\hline I & & I & I & 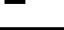 & I & 36 & -0.083 & -0.087 & 41.8460 & 0.139 \\
\hline
\end{tabular}

Fuente: elaboración propia 
Dependent Variable: D(TEMPERATURA, 1)

Method: Least Squares

Date: 08/24/16 Time: 21:09

Sample (adjusted): 18852015

Included observations: 131 after adjustments

Convergence achieved after 8 iterations

MA Backcast: 18831884

\begin{tabular}{|c|c|c|c|c|}
\hline Variable & Coefficient & Std. Error & t-Statistic & Prob. \\
\hline C & 0.006303 & 0.002173 & 2.90024 & 0.0044 \\
\hline $\operatorname{AR}(32)$ & -0.215325 & 0.081230 & -2.650808 & 0.0091 \\
\hline $\operatorname{AR}(34)$ & -0.174549 & 0.080281 & -2.174236 & 0.0315 \\
\hline $\mathrm{MA}(2)$ & -0.553907 & 0.074275 & -7.457513 & 0.0000 \\
\hline R-squared & 0.175266 & \multicolumn{2}{|c|}{ Mean dependent var } & 0.008321 \\
\hline Adjusted R-squared & 0.155784 & \multicolumn{2}{|c|}{ S.D. dependent var } & 0.081864 \\
\hline S.E. of regression & 0.075218 & \multicolumn{2}{|c|}{ Akaike info criterion } & -2.306795 \\
\hline Sum squared resid & 0.718533 & \multicolumn{2}{|c|}{ Schwarz criterion } & -2.219002 \\
\hline Log likelihood & 155.0951 & \multicolumn{2}{|c|}{ Hannan-Quinn criter. } & -2.271121 \\
\hline F-statistic & 8.996354 & \multirow{2}{*}{\multicolumn{2}{|c|}{ Durbin-Watson stat }} & 2.094264 \\
\hline Prob(F-statistic) & 0.000019 & & & \\
\hline \multirow[t]{9}{*}{ Inverted AR Roots } & $.97+.09 \mathrm{i}$ & $.97-.09 \mathrm{i}$ & $.93+.27 \mathrm{i}$ & $.93-.27 \mathrm{i}$ \\
\hline & $.86+.44 \mathrm{i}$ & $.86-.44 \mathrm{i}$ & $.76+.60 \mathrm{i}$ & $.76-.60 \mathrm{i}$ \\
\hline & $.63-.73 . \mathrm{i}$ & $.63+.73 \mathrm{i}$ & $.47-.83 \mathrm{i}$ & $.47+.83 \mathrm{i}$ \\
\hline & $.30+.89 \mathrm{i}$ & $.30-.89 \mathrm{i}$ & $.13+.91 \mathrm{i}$ & $.13+.91 \mathrm{i}$ \\
\hline & $.00-.87 \mathrm{i}$ & $.-.00+.87 \mathrm{i}$ & $.-.13-.91 \mathrm{i}$ & $-.13+91 \mathrm{i}$ \\
\hline & $.-.30-.89 \mathrm{i}$ & $.-.30+.89 \mathrm{i}$ & $.-.47-.83 \mathrm{i}$ & $-.47+.83 \mathrm{i}$ \\
\hline & $-.63+.89 \mathrm{i}$ & $.-.63-.73 \mathrm{i}$ & $-.76+.60 \mathrm{i}$ & $.-.76-.60 \mathrm{i}$ \\
\hline & $--.86+.44 \mathrm{i}$ & $.-86-.44 \mathrm{i}$ & $.-.93-.27 \mathrm{i}$ & $-.93+.27 \mathrm{i}$ \\
\hline & $.-.97-.09 \mathrm{i}$ & $.-.97+.09 \mathrm{i}$ & & \\
\hline Inverted MA Roots & 0.74 & -0.74 & & \\
\hline
\end{tabular}


Todos los parámetros son significativos (Akaike: -2.309, Durbin-Watson: 2.162). Aunado a esto, el modelo es invertible. Con el cumplimiento de los anteriores indicadores, el modelo Arima es el más adecuado, sin embargo, es fundamental que cumpla con otros supuestos — que los residuales no estén correlacionados y que sean homocedásticos-. Cuando se cumpla con ellos se podrá hacer proyecciones.

\section{Validación del modelo}

Esta sección consiste en validar la eficiencia de lo modelo, lo que se realiza mediante apartados:

1. Análisis de los coeficientes: el modelo debe cumplir con la estacionaridad e inveritibilidad y que sus parámetros sean estadísticamente significativos, con un P-valor mayor a 0.05 .

2. Bondad de ajuste: el ajuste del modelo debe ser analizado mediante la utilización del $\mathrm{R}^{2}$ ajustado $\mathrm{y}$ el Durbin-Watson.

3. Análisis de los residuales: estos no deben ser incorrelacionados y deben tener varianza constante.

En el correlograma del modelo Arima se puede observar que las autorrelaciones no tienen un decrecimiento suavizado. Esto corrobora que, mediante la primera diferenciación, el modelo tiene estacionaridad.

A través de la prueba de raíces unitarias, la primera diferenciación del modelo presentaría estacionaridad, ya que su P-valor es inferior a 0.05 . Por tanto, hay evidencia estadística para afirmar que no existen raíces unitarias. Por otro lado, el modelo de primera diferenciación es invertible.

- Bondad de ajuste: a partir de que el modelo Arima tiene estacionaridad y es invertible, su R2 ajustado es de 0.1557 . Esto significa que el modelo conserva el $15.57 \%$ de la variabilidad de los datos, es decir, el modelo de series de tiempo explica en un $15.57 \%$ el calentamiento global.

- Por otro lado, el Durbin-Watson mide el grado de autocorrelación entre los residuos correspondientes de cada observación y la anterior. El Durbin-Watson del modelo es de 2.09 2, lo cual indica la incorrelación de los residuales del modelo Arima.

- Análisis de los residuales: como se vio anteriormente, el Durbin-Watson del modelo IV indica que sus residuales están incorrelacionados. Esta afirmación se puede corroborar mediante la prueba de Breusch-Godfrey.

En la tabla 3 se puede ver que el F estadístico y la Chi-cuadrada se encuentran por arriba de 0.05 . En consecuencia, los residuales son incorrelacionados, es decir, no hay suficiente información en los últimos 36 retardos para que pueda mejorar el modelo Arima.

Por otro lado, en la tabla 5 se puede observar que a través de la prueba $\mathrm{ARCH}$ de heterocedasticidad, el modelo Arima presenta probabilidades en F y por arriba de 0.05 . Por consiguiente, existe evidencia estadística para asegurar que los residuales del modelo IV son homocedasticos, es decir, tienen varianza controlada.

En el histograma de normalidad (tabla 4), se puede ver que los residuales se aproximan a una normal, lo que se puede corroborar mediante P-valor: si este es inferior a 0.05, no habría normalidad en los residuales. En el modelo Arima, el P-valor es superior a 0.05, en consecuencia, sus residuales se aproximan a una normal.

En síntesis, el modelo Arima de series de tiempo cuenta con los elementos necesarios para poder pronosticar el calentamiento global, ya que tiene estacionaridad y es invertible. Asimismo, sus residuales están incorrelacionados, son homocedasticos y se aproximan a una normal. 
Tabla 3. Análisis de los residuales del modelo Arima

\begin{tabular}{|c|c|c|c|c|}
\hline \multicolumn{5}{|c|}{ Breusch-Godfrey Sarial Correlation LM Test: } \\
\hline F-statistic & 0.831664 & Prob. $\mathrm{F}(36,91)$ & 0.729 & \\
\hline Obs*R-squared & 32.42926 & Prob. Chi-Square(36) & 0.6392 & \\
\hline \multicolumn{5}{|c|}{ Dependent Variabgle: REDID } \\
\hline \multicolumn{5}{|c|}{ Sample: 18852015} \\
\hline \multicolumn{5}{|c|}{ Included observations: 131} \\
\hline \multicolumn{5}{|c|}{ Presample missing value lagged residuals ser to zero. } \\
\hline Variable & Coefficient & Std. Error & $t$-Statistic & Prob. \\
\hline$\overline{\mathrm{C}}$ & -0.000533 & $\overline{0.002549}$ & -0.208942 & 0.8350 \\
\hline $\mathrm{AR}(32)$ & -0.043284 & 0.151649 & -0.285426 & 0.7760 \\
\hline $\operatorname{AR}(34)$ & -0.076090 & 0.149455 & -0.509117 & 0.6119 \\
\hline MA(2) & -0.758406 & 0.318855 & -2.378530 & 0.0195 \\
\hline RESID $(-1)$ & 0.047604 & 0.103386 & 0.460450 & 0.6463 \\
\hline $\operatorname{RESID}(-2)$ & 0.848816 & 0.322247 & 2.634053 & 0.0099 \\
\hline RESID(-3) & -0.042064 & 0.102968 & -0.408517 & 0.6839 \\
\hline RESID $(-4)$ & 0.430344 & 0.201121 & 2.139727 & 0.0351 \\
\hline RESID(-5) & 0.179413 & 0.102701 & 1.746944 & 0.0840 \\
\hline RESID (-6) & 0.014014 & 0.139892 & 0.100178 & 0.9204 \\
\hline RESID(-7) & 0.121037 & 0.106796 & 1.133345 & 0.2600 \\
\hline RESID(-8) & 0.105230 & 0.116751 & 0.901316 & 0.3698 \\
\hline RESID(-9) & -0.121094 & 0.107395 & -1.127558 & 0.2625 \\
\hline RESID(-10) & 0.161863 & 0.112567 & 1.437927 & 0.1539 \\
\hline RESID(-11) & -0.047989 & 0.108279 & -0.443197 & 0.6587 \\
\hline RESID(-12) & 0.012838 & 0.111165 & 0.115483 & 0.9083 \\
\hline RESID(-13) & 0.107445 & 0.108043 & 0.994466 & 0.3226 \\
\hline RESID(-14) & 0.024768 & 0.109832 & 0.225510 & 0.8221 \\
\hline RESID(-15) & -0.012428 & 0.108672 & -0.114361 & 0.9092 \\
\hline RESID(-16) & 0.167633 & 0.111571 & 1.502479 & 0.1364 \\
\hline RESID(-17) & 0.080186 & 0.110675 & 0.755035 & 0.4522 \\
\hline RESID(-18) & 0.001891 & 0.116314 & 0.016262 & 0.9871 \\
\hline RESID(-19) & 0.080186 & 0.113853 & 0.704293 & 0.4830 \\
\hline RESID(-20) & 0.012463 & 0.122233 & 0.101962 & 0.9190 \\
\hline RESID(-21) & -0.073365 & 0.123172 & -0.595632 & 0.5529 \\
\hline RESID(-22) & 0.031081 & 0.123916 & 0.250825 & 0.8025 \\
\hline RESID(-23) & -0.028180 & 0.127671 & -0.233556 & 0.8159 \\
\hline RESID(-24) & -0.043905 & 0.126784 & -0.346301 & 0.7299 \\
\hline RESID(-25) & 0.044356 & 0.130373 & 0.340228 & 0.7345 \\
\hline RESID(-26) & -0.033435 & 0.132605 & -0.252139 & 0.8015 \\
\hline RESID(-27) & -0.110207 & 0.133456 & -0.825790 & 0.4111 \\
\hline RESID(-28) & -0.065362 & 0.133700 & -0.488872 & 0.6261 \\
\hline RESID(-29) & 0.053100 & 0.134021 & 0.396207 & 0.6929 \\
\hline RESID(-30) & -0.043512 & 0.136550 & -0.318654 & 0.7507 \\
\hline RESID(-31) & -0.090695 & 0.133754 & -0.677756 & 0.4996 \\
\hline RESID(-32) & 0.050927 & 0.198825 & 0.256139 & 0.7984 \\
\hline RESID(-33) & 0.087456 & 0.127413 & 0.686401 & 0.4942 \\
\hline RESID(-34) & 0.022657 & 0.194077 & 0.116745 & 0.9073 \\
\hline RESID(-35) & -0.047765 & 0.121657 & -0.392622 & 0.6955 \\
\hline $\operatorname{RESID}(-36)$ & -0.081654 & 0.123434 & -0.661519 & 0.5100 \\
\hline R-squared & 0.247552 & Mean dependent var & & 0.000250 \\
\hline Adjusted R-squared & -0.074926 & S.D. dependent var & & 0.074345 \\
\hline S.E. of regression & 0.077079 & Akaike info criterion & & -2.041611 \\
\hline Sum squared resid & 0.540653 & Schwarz criterion & & -1.163688 \\
\hline Log likelihood & 173.725500 & Hannan-Quinn criter. & & -1.684871 \\
\hline F-statistic & 0.767655 & Durbin-Watson stat & & 1.968384 \\
\hline Prob(F-statistic) & 0.820883 & & & \\
\hline
\end{tabular}

Fuente: elaboración propia 
Tabla 5. Homocedasticidad y normalidad los residuales del modelo IV

\begin{tabular}{|c|c|c|c|c|}
\hline \multicolumn{5}{|c|}{ Heteroskedasticity Test: ARCH } \\
\hline F-statistic & 1.078829 & & Prob.F(1,128) & 0.3009 \\
\hline Obs*R-squared & 1.086528 & & Prob.Chi-Square(1) & 0.2972 \\
\hline \multicolumn{5}{|c|}{$\begin{array}{l}\text { Test Equation: } \\
\text { Dependent Variable: RESID^2 } \\
\text { Method: Least Squares } \\
\text { Date: } 08 / 25 / 16 \text { Time: } 11: 40 \\
\text { Incuded observations: } 130 \text { after adjustments }\end{array}$} \\
\hline Variable & Coeficient & Std. Error & $t$-Statistic & Prob. \\
\hline $\begin{array}{l}\mathrm{C} \\
\operatorname{RESID}^{\wedge} 2(-1)\end{array}$ & $\begin{array}{l}0.0049 \\
0.0912\end{array}$ & $\begin{array}{l}0.0009 \\
0.0088\end{array}$ & $\begin{array}{l}5.5822 \\
1.0387\end{array}$ & $\begin{array}{l}0.0000 \\
0.3009\end{array}$ \\
\hline $\begin{array}{l}\text { R-squared } \\
\text { Adjusted R-squared } \\
\text { S.E. of regression } \\
\text { Sum squared resid } \\
\text { Log likelihood } \\
\text { F-statistic } \\
\text { Prob(F-statistic) }\end{array}$ & $\begin{array}{l}0.008358 \\
0.000611 \\
0.008458 \\
0.009158 \\
463.9837 \\
1.078829 \\
0.300918\end{array}$ & $\begin{array}{l}\text { Mean de } \\
\text { S.D. dep } \\
\text { Akaike i } \\
\text { Schwarz } \\
\text { Hannan } \\
\text { Durbin }\end{array}$ & $\begin{array}{l}\text { ependent var } \\
\text { pendent var } \\
\text { info criterion } \\
\text { criterion } \\
\text { - Quinn criter } \\
\text { - Watson stat }\end{array}$ & $\begin{array}{l}0.00544 \\
0.00846 \\
-6.69206 \\
-6.64794 \\
-6.67413 \\
2.03577\end{array}$ \\
\hline
\end{tabular}

Fuente: elaboración propia

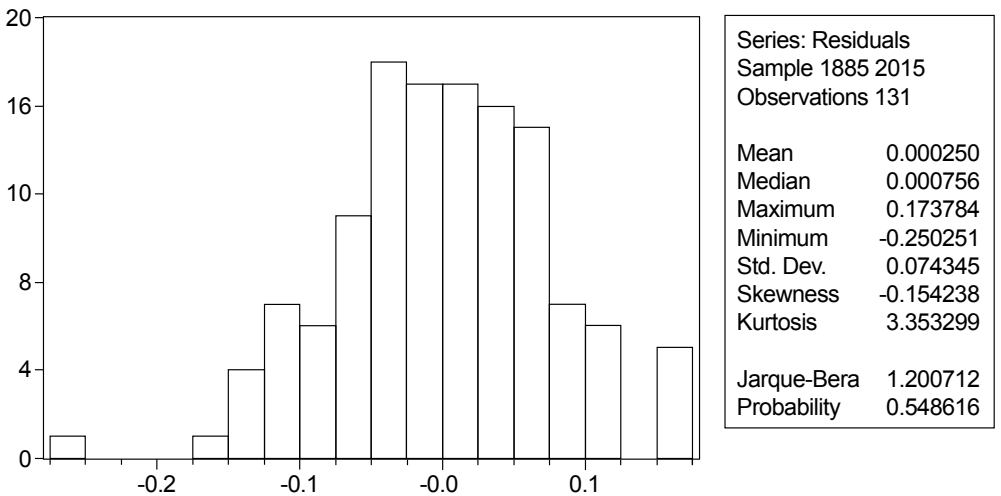

Tabla 4. Histograma de normalidad

Fuente: elaboración propia

\section{Proyecciones}

Para hacer las proyecciones de cualquier modelo es fundamental que se analicen los siguientes conceptos:

- Sesgo de proporción: describe la magnitud de equivocación del modelo en estudio.

- Variancia de proporción: puntualiza la diferenciación de la varianza generada por el modelo en estudio con la varianza de la serie de tiempo original.

- Covarianza de proporción: mide la magnitud de lo no sistemático de la serie de tiempo que explica el modelo.

- Coeficiente de Theil: resume qué tan bueno es el modelo para pronosticar. Toma valores entre 0 y 1 . 
- 0 es buen ajuste.

- 1 es mal ajuste.

El mejor modelo, es aquel que cumpla con lo siguiente:

- Sesgo de proporción 0

- Variancia de proporción 0

- Covarianza de proporción 1

- Coeficiente de Theil 0

Con base en lo anterior, en la figura 2 se puede observar lo siguiente:

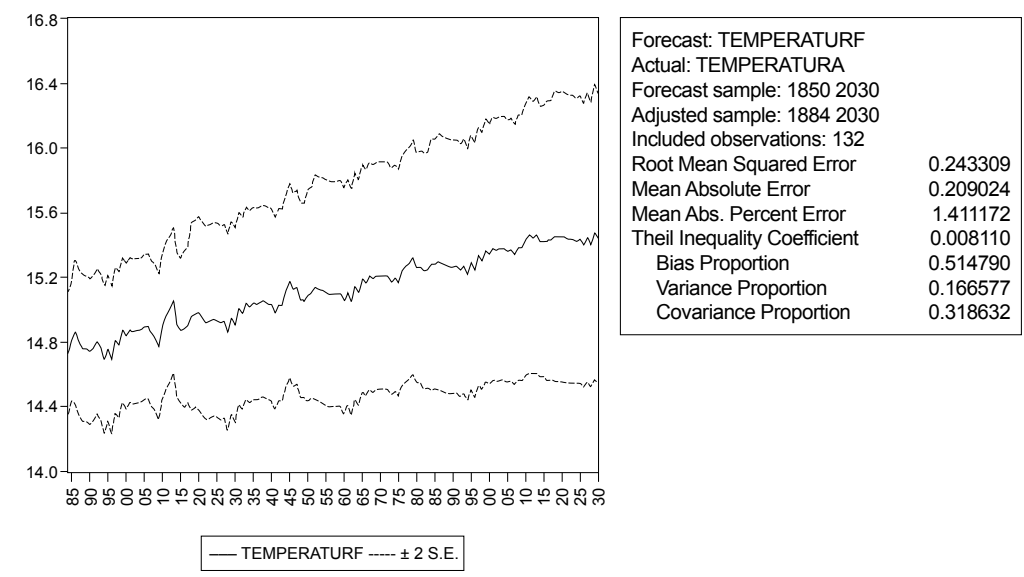

Figura 2. Predicción de la temperatura promedio del modelo IV

Fuente: elaboración propia

Si se realizan proyecciones con el modelo IV, sus indicadores serían los siguientes:

- Sesgo de proporción $=0.5147$

- Variancia de proporción $=0.1665$

- Covarianza de proporción 0.3186 --.-------- (regular)

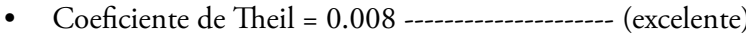

Con este resultado se podría decir que el modelo es bueno para hacer predicciones sobre el calentamiento global.

A través de esta evaluación, podríamos decir lo siguiente: con un nivel de significancia del $95 \%$, un margen de error del $5 \%$ y un R2 ajustado del $15.57 \%$, la temperatura promedio del planeta oscilaría entre 14.55 y $16.33^{\circ} \mathrm{C}$, pues para ese año se espera que la temperatura promedio del planeta fuese en promedio de $15.44^{\circ} \mathrm{C}$.

Por otro lado, en el modelo se puede ver que en el año 1850 la temperatura era de $14.71^{\circ} \mathrm{C}$ y que en la actualidad (2016) sería de $15.43^{\circ} \mathrm{C}$, con lo cual se diría que la temperatura promedio del planeta durante 166 años se ha incrementado en $0.72^{\circ} \mathrm{C}$.

En la figura 3 se puede observar que en el año 1963 la temperatura promedio del planeta presentó fuertes aumentos: era de $15.14^{\circ} \mathrm{C}$. Si se compara con la de la actualidad $\left(15.43^{\circ} \mathrm{C}\right)$, en 53 años la temperatura promedio se ha incrementado en $0.29^{\circ} \mathrm{C}$. 


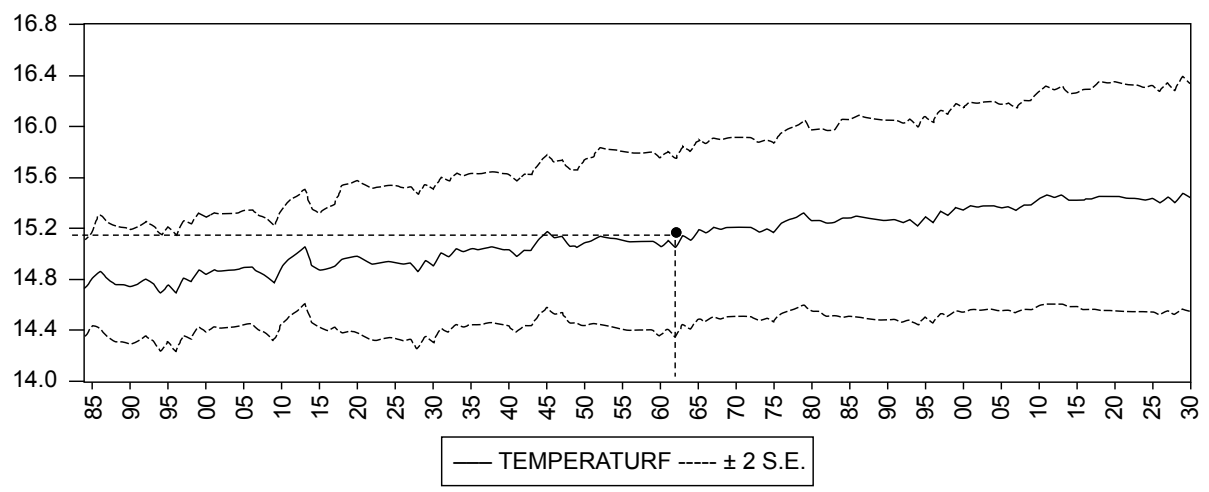

Figura 3. Comportamiento de la temperatura promedio

Fuente: elaboración propia

Por otro lado, en la figura 4 se observa que en el año 1995 la temperatura promedio del planeta se ha salido fuertemente de sus intervalos. Ello indica que hay sobreexplotación de los recursos, lo cual ha provocado el sobrecalentamiento global, y este a su vez a dado lugar al efecto invernadero.

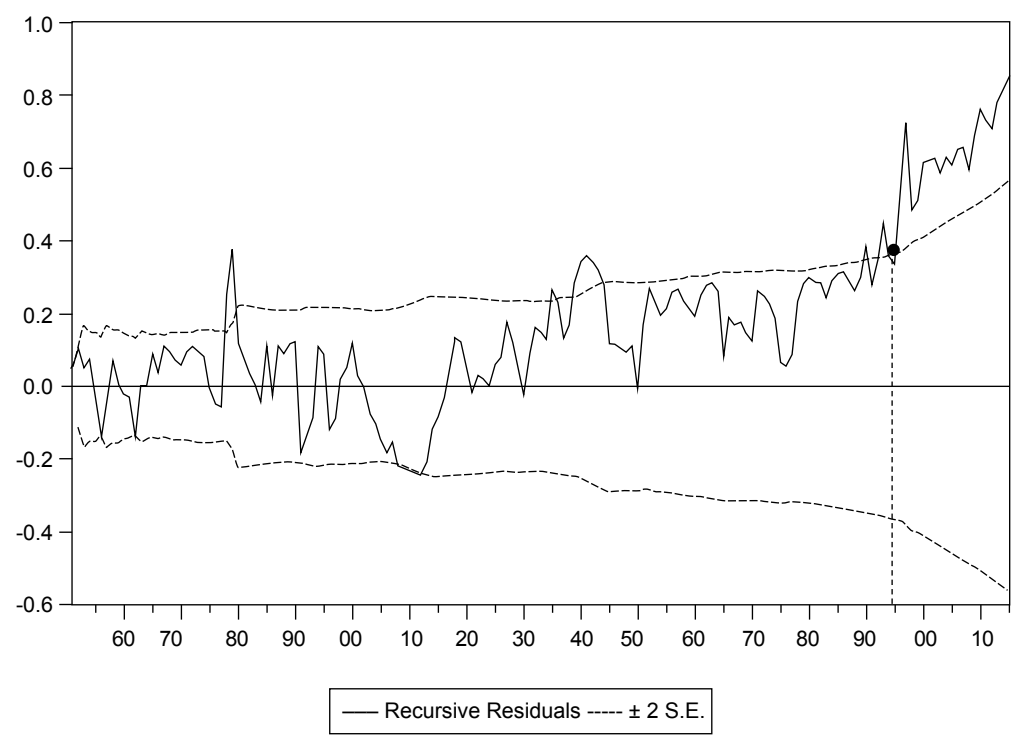

Figura 4. Descompensación de la temperatura promedio Fuente: elaboración propia 


\section{Conclusiones}

Este modelo permite tener un panorama general de las variaciones que ha experimentado el fenómeno del cambio climático, como un fenómeno aleatorio, con un manejo de incertidumbre.

Con este modelo probabilístico se pudo demostrar que la temperatura promedio del planeta se ha incrementado en $0.72^{\circ} \mathrm{C}$ en 165 ańos, de 1850 a diciembre de 2016, muy cercano a lo estipulado por el IPCC, con sus modelos estadísticos, que es de $0.74^{\circ} \mathrm{C}$.

Adicionalmente, desde 1963, la temperatura promedio del planeta ha tenido un comportamiento creciente, tanto que se ha incrementado en $0.29 \mathrm{C}^{\circ}$, sin embargo, es hasta 1996 cuando los efectos del incremento de la temperatura promedio han generado efectos negativos sobre el planeta, ya que a partir de esa fecha la temperatura promedio ha oscilado por arriba de los intervalos establecidos por el modelo, posiblemente esto se debe a la sobre explotación de los recursos naturales y a las altas emisiones de gases de efecto invernadero (principalmente el dióxido de carbono y el metano) como resultado de la actividad humana.

Con este modelo de series de tiempo se pudo pronosticar la temperatura promedio del planeta para el año 2030 , la cual va a oscilar entre los 14.55 y $16.33^{\circ} \mathrm{C}$. La temperatura promedio es de $15.44^{\circ} \mathrm{C}$. A modo de comparación, la temperatura en el año 1850 era de $14.71^{\circ} \mathrm{C}$ y en 2016 fue de $15.43^{\circ} \mathrm{C}$. Contando con que las actividades humanas no cambien — pero podemos asegurar que las actividades humanas se irán incrementando, aun exponencialmente-, el modelo nos da un escenario de hasta $16.33^{\circ} \mathrm{C}$.

En este contexto, se puede decir que el acelerado crecimiento que ha experimentado la temperatura promedio del planeta a partir del 1963, y que se ha agudizado desde 1996, es resultado de la forma en que han crecido las economías de todo el mundo, las cuales se han sustentado en la sobreexplotación de los recursos naturales.

El medio ambiente, los modelos matemáticos estadísticos y la presente propuesta sobre un modelo matemático probabilístico contribuyen a comprender los fenómenos ambientales, a medir los resultados y a tomar decisiones sobre ellos. Para los ambientalistas, estos modelos no son la solución definitiva sobre un fenómeno, sin embargo, pueden constituir una buena herramienta para conocer los procesos medioambientales y, si es necesario, intervenir en ellos.

\section{Referencias}

Arya, J. y Lardner, R. (2009). Matemáticas aplicadas a la administración y la economía. s. 1.: Prentice Hall.

Caballero, M., Lozano, S. y Ortega, B. (2007). Efecto invernadero, calentamiento global y cambio climático: una perspectiva desde las Ciencias de la Tierra. México: Revista Digital Universitaria.

Collazo, A. (2010). Apuntes sobre el método simplex de programación lineal. San Juan: Universidad de Puerto Rico.

Costeau, J. (1992). Impacto ambiental. El planeta herido. Recuperado de http://assets.mheducation.es/bcv/guide/ capitulo/8448167155.pdf

Eschenhagan, M. (2006). Las cumbres ambientales internacionales y la educación ambiental. OASIS, (12), 39-76.

Ferrero, J. (20 10). Del calentamiento global al cambio climático ¿una teoría más? Recuperado de file://C:/Users/

DELL/Downloads/DialnetDelCalentamientoGlobalAlCambioClimaticoUnaTeoriaMa-3800498\%20

(1).pdf

García, J. y Maheut, J. (2015). Modelado y resolución de problemas de organización industrial mediante programación

matemática lineal (modelos y métodos de investigación de operaciones. Procedimientos para pensar). s. 1.: Universidad Politécnica de España. 
Gitay, H., Suárez, A. y Watson, R. (2002). Cambio climático y biodiversidad. s. l.: Grupo Intergubernamental de Expertos Sobre el Cambio Climático.

Green Facts. (2007) Página web. Recuperado en abril del 2017, de https://www.greenfacts.org/es/dosieres/ cambio-climatico.htm

Lopéz, C. (2012). Los países emergentes ante el reto de la sostenibilidad. Recuperado de http://www.profesiones.org/ var/plain/storage/original/application/5bee201e55097ef4e954620cfdb07c87.pdf

Ramos, A., Sánchez, P., Ferrer, J. y Barquín, J. (2010). Modelos matemáticos de optimización. Publicación Técnica, (1).

Rosales, R. (2009). Metodología Box-Jenkins. Bogotá: Universidad de los Andes.

Villavicencia, J. (2008). Introducción a series de tiempo. Recuperado de http://www.estadisticas.gobierno.pr/iepr/ LinkClick.aspx?fileticket=4_BxecUaZmg\%3D 